Gynäkologe 2014 · 47:8-8

DOI 10.1007/s00129-013-3198-7

Online publiziert: 6. Dezember 2013

(c) Springer-Verlag Berlin Heidelberg 2013

\section{W. Janni ${ }^{1} \cdot 0$. Ortmann $^{2}$}

${ }^{1}$ Frauenklinik, Universitätsklinikum Ulm

${ }^{2}$ Caritas-Krankenhaus St. Josef, Klinik für Frauenheilkunde und Geburtshilfe der Universität Regensburg

\title{
Multimodale Therapie des Uterus myomatosus
}

Liebe Kolleginnen und Kollegen,

„Schon wieder Myome?" - werden Sie sich vielleicht gefragt haben, als Sie dieses Themenheft zur Hand genommen haben.

Ja, schon wieder. Denn Uterusmyome gehören zur frauenärztlichen Tätigkeit, wie der Umgang mit Hormonen, Schwangerschaft und Malignomen. Aufgrund ihrer Häufigkeit und der Komplexität im therapeutischen Umgang fordern sie uns regelmäßig heraus:

- Myome sind die häufigsten soliden Tumore des weiblichen Genitaltraktes.

- Zwischen 20 und 40\% der Frauen im reproduktiven Alter entwickeln Myome.

- In Europa haben etwa 24 Mio. Frauen Myome.

- 20-30\% der Hysterektomien erfolgen zur Behandlung von Myomen.

- Eine aktuelle amerikanische Studie geht von direkten Gesundheitskosten (Chirurgie, stationäre Krankenhausaufenthalte, Arzneimittel) von 4-9 Mrd. US-Dollar jährlich aus.

Während diese Aussagen zwar die Bedeutung des Krankheitsbildes unterstreichen, aber keine Neuigkeiten enthalten, ändern sich die Möglichkeiten zur interventionellen Therapie ständig. Kaum eine benigne Erkrankung in unserem Fachgebiet hat in den vergangenen 10 Jahren so viele Innovationen erfahren wie der Uterusmyome.

Grund genug für eine Standortbestimmung, meinen wir. Wir versuchen, in diesem Heft einen ausgewogenen Überblick über den aktuellen Stand aller Therapieoptionen zu geben, auch über deren Kon- sequenzen und Risiken. Wir sind zuversichtlich, dass Sie aufgrund der Häufigkeit des Krankheitsbildes das gewonnene Wissen hoffentlich sehr bald anwenden können!

\section{》) Die Optionen für eine interventionelle Therapie ändern sich ständig}

Wir wünschen Ihnen eine interessante Lektüre und viel Erfolg bei der Anwendung der neu gewonnenen Erkenntnisse, für Ihre Patientinnen und für Sie,

Ihre

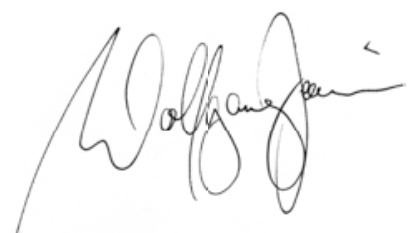

Prof. Dr. Wolfgang Janni

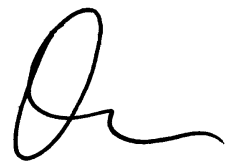

Prof. Dr. Olaf Ortmann

\section{Korrespondenzadresse}

Prof. Dr. W. Janni

Frauenklinik, Universitätsklinikum Ulm Prittwitzstr. 43, 89075 Ulm

wolfgang.janni@uniklinik-ulm.de

Interessenkonflikt. W. Janni und O. Ortmann geben an, dass kein Interessenkonflikt besteht. 